\title{
FORMATION OF THE UPSTREAM REGION IN HV GAS BLAST INTERRUPTERS WITH SYNCHRONOUS GAS INJECTION
}

\author{
N.K. Kurakina ${ }^{a, b, *}$, V.YA. Frolov ${ }^{a}$, E.N. TONKOnOGOV ${ }^{a}$ \\ ${ }^{a}$ Peter the Great Saint-Petersburg Polytechnic University, Polytechnicheskaya 29, St.-Petersburg 195251, Russia \\ ${ }^{b}$ Institute for Electrophysics and Electric Power of Russian Academy of Sciences, Dvortsovaya nab., 18, \\ St.-Petersburg, 191186, Russia \\ * nkuriee@gmail.com
}

\begin{abstract}
The problem of a synchronous gas injection at the vicinity of current zero for high voltage gas blast circuit breakers (GCB) is discussed. The basic aim of the investigation is to improve the efficiency of arc quenching in GCB by increasing interruption ability without changes in drive parameters. The paper reviews the upstream region influence on the interruption capability, gas injection - mass flow interactions at the region between electrical contact and nozzle throat of GCB. Numerical simulation is used to calculate gas-dynamics parameters at the upstream region of mono-flow GCB. The mass discharged as a result of the synchronous gas injection can rapidly modify both the density and pressure at the nozzle throat, regulate flow velocity in the research region. The selected method of the gas injection effects the breaking performance in the arc quenching device strongly. Some calculation results are presented.
\end{abstract}

Keywords: high-voltage gas blast interrupters, air blast circut breakers, interruption capability, arc quenching, synchronous gas injection, gas-dynamics, numerical simulation, OpenFOAM.

\section{Introduction}

The power growth in electrical networks imposes the continued efforts to improve the performance of the circuit breakers. According to the Kioto protocol, agreed in 1997, the use of sulfur hexafluoride in electric power transmission and distribution systems must to be minimized despite its high efficiency. The search of alternative gases of natural origin and the appliance of dry air, $\mathrm{CO}_{2}$, and $\mathrm{N}_{2}$ still remain an urgent problem $[1,2]$. It is therefore useful to develop of GCB which are advanced to a high performance level. Today, the drive mechanics has a particular impact on the efficiency of the synchronous GCB. The high proportion failures (52-72\% of the overall failure rates [3]) are attributed to the drivers. Furthermore, the complicated drives construction contradicts the modern requirements of resource-intensiveness and the high criteria for operation according to the international standards [4].

The present study is aimed at increasing the interruption capability of the mono-flow gas arc quenching device without a complication of the drive constructions with an alternative to $\mathrm{SF}_{6}$ medium. An example of such medium is dry air. It is proposed to influence the switching arc parameters by a short-time gas pulse of high pressure at the vicinity of current zero. The main reason of electrical breakdown post current zero is a very hot remnant of the arc channel between the contacts. The intensification of the cold gas flow removes the surplus of energy of the decaying arc column as well as the complexity of the pressure, mass, and velocity interchanges (due to the impulse gas injection) increases the turbulent mixing to enable a successful arc quenching.

This article presents the model validation, the gas dynamics parameters (mass flow rate, pressure and etc) in the upstream and nozzle throat regions for cold and hot models obtained by means of the free open-source package OpenFOAM for the numerical simulation.

\section{Model device and methods}

The model of the mono-flow gas arc quenching device (figure 1) was chosen by the authors due to the accessibility of experimental data, necessary for the conclusions in this work and the subsequent experimental studies.

Reference [5] identified which design parameters were dominant in establishing the optimum rate of rise of recovery voltage performance $(R R R V)$. According to these results, the nozzle size and shape of the model device (figure 1 ) were chosen on the assumption $L / D=1$, where $L$ is the distance between the fixed contact and the critical section of the nozzle, and $D$ is the diameter of the nozzle. The working gas (air) of pressure $p_{0}$ is fed into the upstream area through the inlet. The gas flow interaction builds the upstream region.

There is a general relationship in the switchgear literature $[6,7]$ that the interruption capability of GCB increases significantly with gas pressure growth and reduces with increasing current. The logarithmic relationship is shown in figure 2 of the parameter $R V^{*}=(\mathrm{d} U / \mathrm{d} t) / p_{c r}$, where $p_{c r}=p / 2$, versus the rate of 


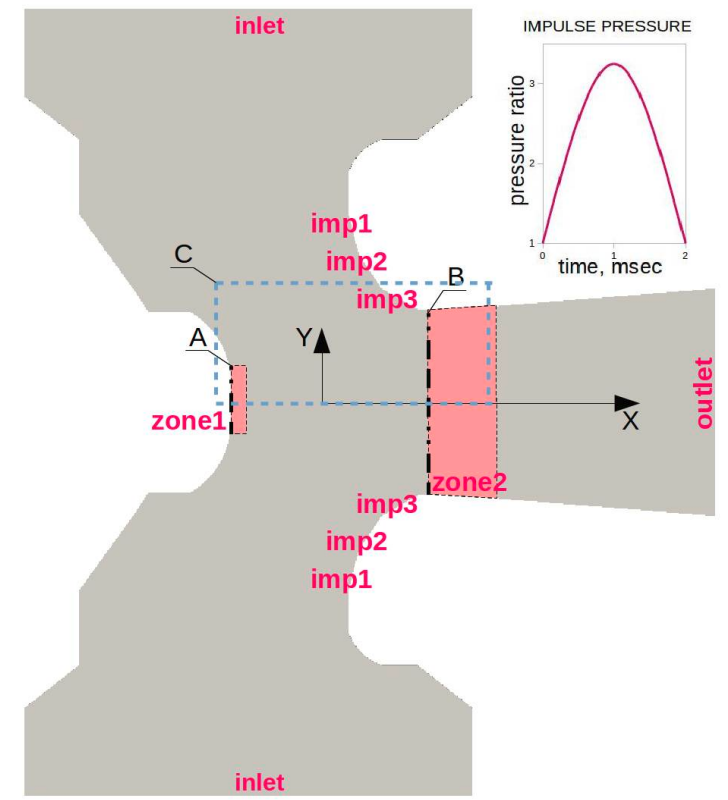

Figure 1. The model of the mono-flow gas blast arc quenching device.

change of the current $\mathrm{d} I / \mathrm{d} t$ for air GCB. The data was obtained in experiments by the authors in Ref. [5, 8$10]$ at various values of upstream pressures and nozzles design constructions.

The analysis of the curves in figure 2 shows the general trend of interaction ability for air GCB. Yellow (1) and blue (2) curves were plotted for double flow arc quenching systems [10]. Their interrupting ability is by a factor of 1.5 higher $[6,7]$ than that of mono-flow devices, see curves (3), (4), (5). Yellow dashed line $\left(1^{*}\right)$ denotes the "actual" location of the curve (1) after dividing by the over-coefficient 1.5. The arching time in the experiment [10] was $1.5 \mathrm{~ms}$. This means that the active arc-gas interaction after current zero took not more than $1 \mathrm{~ms}$. The comparison of mono-flow systems and a synchronous commutation shows that a successful commutation can be reached by using a short-time gas pulse of high pressure with a duration of about $1.5-2 \mathrm{~ms}$ in the interrupting ability of the GCB.

It is suggested, therefore, to deliver a short-time powerful gas impulse of pressure $p$ at the vicinity of current zero by means of an injector (imp1, imp2 or imp3) with a diameter $d$ (here $D / d=4.5$ ). The gas pulse shape and duration (taken as $2 \mathrm{~ms}$ as a first approximation) are presented in figure 1 . The zone 1 is a region of stagnation. It appears with the increase in pressure and the reduction of velocity near the contact. The most probable position of the arc remnant destruction is marked as zone 2. Zones 1 and 2 are the regions of our special interest. The injection pressure $p$ was chosen from the relationship $p / p_{0}=3$ [8].

The gas-dynamics parameters (pressure rate, flow velocity and mass flux) are simulated with the free

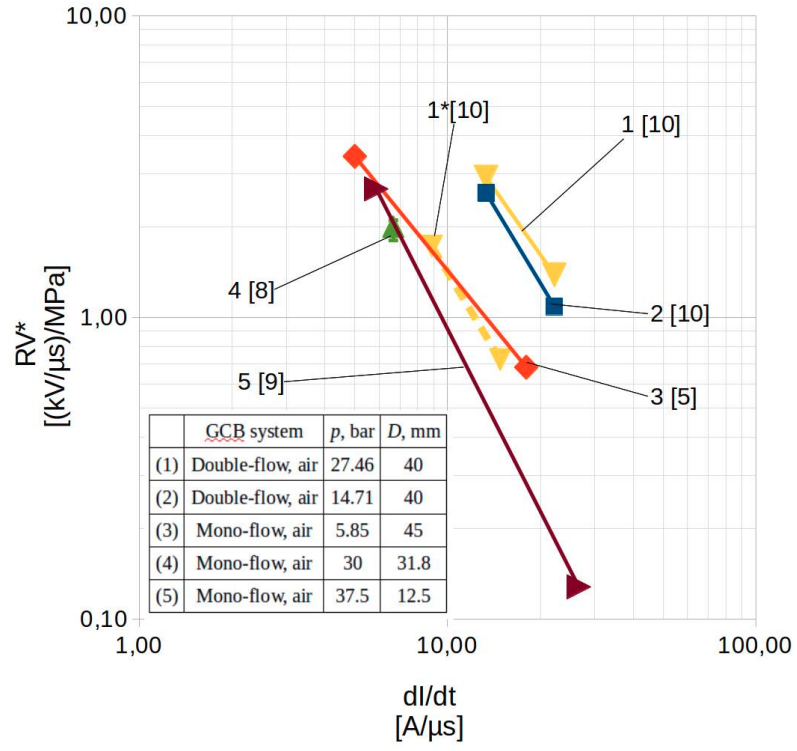

Figure 2. Normalized parameter $R V^{*}$ as a function of $d I / d t$.

open-source package OpenFOAM by adopting a twodimensional model and a continuous medium approximation. The conservation laws of mass, momentum and energy complemented by the state equation serve as a basis for the description of the compressed gas flow with initial supersonic speed in similar systems. The method was described and verified in $[11,12]$ of the experimental stand $[13,14]$, where the calculated results agreed well with experimental data and dependences.

\section{Cold gas flow phenomena with synchronous gas injection}

The interplay of the gas-dynamics parameters has the major contribution to the interruption ability of GCB. The knowledge of the cold gas flow phenomena helps to understand better the nature of the arc-gas correlations and to choose the optimum position for the injection, which is important since it governs the efficiency of the arc quenching.

Figure 3 presents a comparative analysis of the normalized mass flux $Q / Q_{0}$ versus time for zone 1 of the cold model in relation to the basic gas flow value with different injection sites (imp1/imp2/imp3). The initial values are the following: $p / p_{0}=3, D / d=4.5$, $L / D=1$.

The plots in figure 3 show that the best injection site is imp3 for the model of mono-flow GCB due to the maximum time-mean value of the mass flux on the considered region.

Figure 4 presents calculation results of the system pressure $\left(p / p_{0}\right)$ with a synchronous injection at the site imp3 along the chamber axis for various times from the pulse beginning. The normalized distance from the contact (A) is defined as $X=x / D$, where $x$ is 


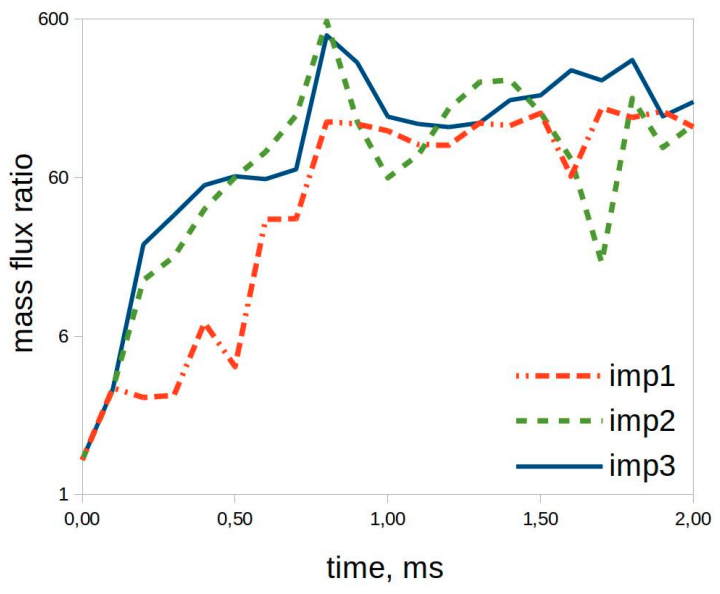

Figure 3. Mass flux ratio $Q / Q_{0}$ in zone 1 for different injectors sites (imp1, imp2, imp3).

the distance along the axis OX of the nozzle channel, and $D$ is the nozzle diameter.

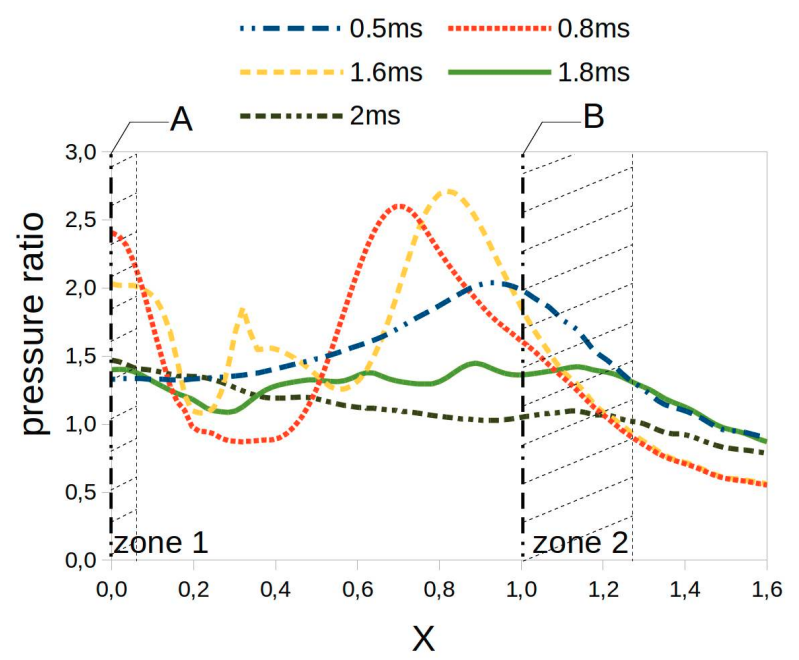

Figure 4. Pressure ratio along OX for various times from injection beginning at imp3.

The injection of high pressure gas begins at time $t$ in the vicinity of current zero. Here, the zero value is conditionally taken (see figure 4). The gas pulse is supplied to the computational domain when the basic stationary flow is reached. A sharp pressure jump occurs in front of the nozzle throat during the period between 0.5 and $0.8 \mathrm{~ms}$ (blue-red curves). Afterwards, the reflected shock wave begins to propagate to zone 1 near the contact and is reflected on it, intensely affecting the stagnation points. The pressure variation is determined by the interaction of the waves and their successive reflection from the fixed elements of the arc quenching chamber in the time interval (0.8-1.6) $\mathrm{ms}$ (red-yellow curves). At $t=2 \mathrm{~ms}$ (green-black curves), unsteady decaying waves are observed and the pressure value reduces to the level $p_{0}$, determined by the basic gas flow.

As a result, the directional synchronous gas injec- tion controls the density and pressure differential of the mass discharged. The pressure differential regulates the gas velocity. The maximum effect of a turbulent mixing is achieved. According to the calculation results, the gas pulse intensifies and prolongs mass flow fluctuations for more than $100 \mu \mathrm{s}$. It is instrumental in the recovery performance.

\section{Interactions between the switching arc and the synchronous gas injection}

To study how the decaying plasma column influences the upstream region in the mono-flow air arc quenching device with a synchronous gas injection, a "hot" simulation is used. For this purpose, a region of high temperature, small density, initial pressure and velocity was set at the position of the arc column. These parameters have been calculated in a previous simulation.

It is known that the arc diameter is proportional to the square root of the current magnitude. The diameter of the hot arc remnant $d_{a}$ at current zero does not exceed $1 \mathrm{~mm}$ for the rate of change of current $\mathrm{d} I / \mathrm{d} t=24 \mathrm{~A} / \mathrm{\mu s}$ [6]. The plasma temperature varies from $2000 \mathrm{~K}$ on the thermal boundary layer up to $10000 \mathrm{~K}$ on the axis [6]. In the simulation model, the bulk temperature of the arc and the diameter were taken respectively $T=5000 \mathrm{~K}$ and $d_{a}=4 \mathrm{~mm}$ near the contact (A) as a first approximation, taking into account the initial interaction between the injection and the thermal boundary layer at 400-600 $\mu$ s before current zero (see below).

Calculation results of the deformation of the thermal boundary layer for the region $\mathrm{C}$ (see figure 1) are presented in figure 5 . The temperature distribution with a contour line of $T=2000 \mathrm{~K}$ defines the thermal boundary layer in the model. To trace the dynamics of the injection influence on the thermal boundary layer, the curves are plotted for various times from the beginning of the injection: $t_{0}=0 \mathrm{~ms}-$ start of the injection (yellow line), $t_{1}=0.35 \mathrm{~ms}-$ the initial time of active interaction between the front of the gas pulse and the arc boundary layer (blue line), $t_{2}=t_{1}+100 \mu \mathrm{s}$ (red line), $t_{3}=t_{2}+50 \mu$ s (black line).

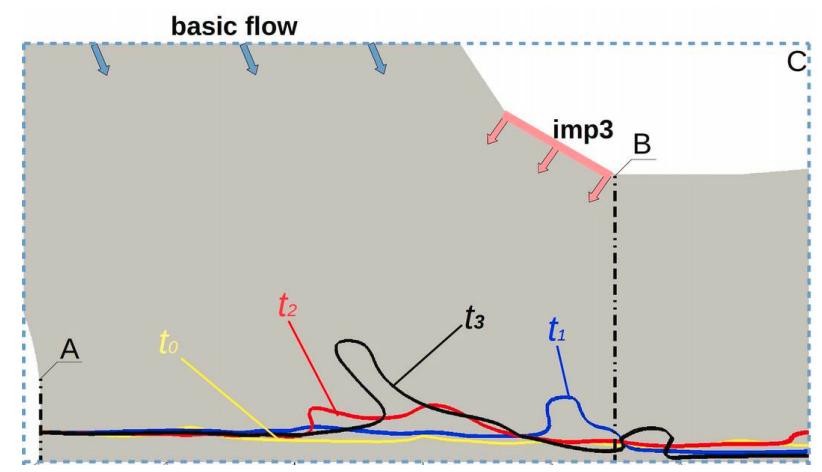

Figure 5. Thermal boundary layer deformation for various times from injection beginning. 
Figure 5 confirms that the synchronous gas injection has a deforming effect on the boundary layer for the "hot" model simulation. The general deformation is defined by the mass connecting to the area. A boundary layer displacement and an increase of the interaction cross-section are observed, providing a turbulent mixing at the upstream region. This would cause a development of the interruption ability and efficiency of the arc quenching.

\section{Conclusions}

The influence of the synchronous gas injection on the gas-dynamics parameters in the HV mono-flow GCB are investigated by cold and hot models simulations. The synchronous gas injection changes the initial gas-dynamics characteristics (density, pressure, velocity, and mass flow rate), deforms the arc thermal boundary layer, provides the turbulent mixing in the upstream region at the vicinity of current zero. All of these points are essential for the improvement of the interruption performance of the GCB. The numerical simulation confirms the efficient contribution of the synchronous gas injection to increase the interruption ability of high voltage gas blast circuit breakers.

\section{References}

[1] H. Hama, S. Okabe, R. Buhler, D. Gautschi, A. Girodet, M. Hering, D. Imamovic, K. Juhre, Y. Kieffel, J. Kindersberger, W. Koltunowicz, J. Lopes-Roldan, S. Neuhold, C. Neumann, R. Pietsch, U. Riechert, T. Rokunohe, U. Schichler, and T. Yasuoka. Dry air, $\mathrm{N}_{2}, \mathrm{CO}_{2}$ and $\mathrm{N}_{2} / \mathrm{SF}_{6}$ mixtures for gas-insulated systems. Electra, page 71, Aug 2018.

[2] M. Seeger, R. Smeets, J. Yan, H. Ito, M. Claessens, E. Dullni, L. Falkingham, C. Franck, F. Gentils, W. Hartmann, Y. Kieffel, S. Jia, G. Jones, J. Mantilla, S. Pawar, M. Rabie, P. Robin-Jouan, H. Schellekens, J. Spencer, T. Uchii, X. Li, and S. Yanabu. Recent trends in development of high voltage circuit breakers with $\mathrm{SF}_{6}$ alternative gas. Plasma Physics and Technology, 4(1):8-12, 2017. doi:10.14311/ppt.2017.1.8.

[3] R. Thomas. Controlled switching of high voltage $\mathrm{SF}_{6}$ circuit breakers for fault interruption, 2004. Thesis for the degree of licentiate of engineering. http://webfiles . portal . chalmers.se/et/Lic/ThomasRichardLic.pdf.

[4] International standard IEC 62271-100. High-voltage switchgear and controlgear. Part 100. Alternating current circuit-breakers, 2012.

[5] A. Briggs and L. King. Factors affecting thermal breakdown in gas blast interrupters. IEE Conf. Dev in Design and Performance, London, pages 52-55, Nov 1979.

[6] K. Ragaller. Current Interruption in High Voltage Networks. Moscow, Energoizdat, 1981.

[7] E. Tonkonogov. HV AC Circuit Breakers. Saint Petersburg, Polytechnic University Press, 2015.

[8] A. Briggs. The contribution of axial a radial components of gas velocity to the thermal interruption capability of a gas blast circuit breaker. Proceedings of the 7th International Conference on Gas Discharges and their Applications, UK, London, pages 28-31, 1982.

[9] G. Frind and J. Rich. Recovery speed of axial flow gas blast interrupter: dependence on pressure and di/ dt for air and $\mathrm{SF}_{6}$. IEEE Transactions on Power Apparatus and Systems, PAS 93(5):1675-1684, Sep 1974. doi: 10.1109/TPAS . 1974.293900.

[10] T. Morita, M. Iwashita, and Y. Nitta. A theoretical analysis of dynamic arcs and test results of model synchronous air blast circuit breakers. IEEE Transactions on Power Apparatus and Systems, PAS-97(3):940-949, May 1978. doi:10.1109/TPAS.1978.354567.

[11] N. Kurakina, M. Pinchuk, A. Budin, A. Smirnovsky, and V. Frolov. Numerical simulation using openfoam of gas-dynamics in the discharge chamber with a movable electrode. St. Petersburg Polytechnic University Journal of Engineering Science and Technology, 24(2):69-81, 2018. doi:10.18721/JEST. 240206.

[12] N. Kurakina, M. Pinchuk, A. Budin, and A. Smirnovsky. Analysis of the gas dynamics in the discharge chamber with a movable electrode. Journal of Physics: Conference Series, 1135:012094, Dec 2018. doi:10.1088/1742-6596/1135/1/012094.

[13] A. Budin, M. Pinchuk, V. Leontev, A. Leks, N. Kurakina, A. Kiselev, J. Simakova, and V. Frolov. Experimental stand for investigations of insulator degradation and electrode erosion in high-current breaker. Plasma Physics and Technology, 4(2):120-123, 2017. doi:10.14311/ppt.2017.2.120.

[14] A. Budin, M. Pinchuk, V. Kuznetsov, V. Leontev, and N. Kurakina. An experimental setup for investigation of arc and erosion processes in high-voltage high-current breakers. Instruments and Experimental Techniques, 60(6):837-842, Nov 2017. doi:10.1134/S0020441217060033. 